PROCEEDINGS OF THE

AMERICAN MATHEMATICAL SOCIETY

Volume 137, Number 1, January 2009, Pages 45-50

S 0002-9939(08)09450-1

Article electronically published on July 25, 2008

\title{
PRODUCTS OF BRAUER-SEVERI SURFACES
}

\author{
AMIT HOGADI \\ (Communicated by Ted Chinburg)
}

\begin{abstract}
Let $\left\{P_{i}\right\}_{1 \leq i \leq r}$ and $\left\{Q_{i}\right\}_{1<i<r}$ be two collections of Brauer-Severi surfaces (resp. conics) over a field $k$. We show that the subgroup generated by the $P_{i}$ 's in $\operatorname{Br}(k)$ is the same as the subgroup generated by the $Q_{i}$ 's if and only if $\prod P_{i}$ is birational to $\prod Q_{i}$. Moreover in this case $\prod P_{i}$ and $\prod Q_{i}$ represent the same class in $M(k)$, the Grothendieck ring of $k$-varieties. The converse holds if $\operatorname{char}(k)=0$. Some of the above implications also hold over a general noetherian base scheme.
\end{abstract}

\section{INTRODUCTION}

1.1. Notation. Let $S$ denote a noetherian base scheme. All products, unless otherwise mentioned, will be over $S$. The class of any Brauer-Severi scheme $P$ over $S$ in $B r(S)$ (the Brauer group of $S$ ) will be denoted by $P$ itself. For a collection of Brauer-Severi schemes $\left\{P_{i}\right\}_{i \in I}$ over $S$, the subgroup generated by the $P_{i}$ 's in $\operatorname{Br}(S)$ will be denoted by $\left\langle\left\{P_{i}\right\}_{i \in I}\right\rangle . M(S)$ will denote the Grothendieck ring of finite type $S$-schemes (see Section 2).

All schemes considered will be noetherian. By a closed subscheme we will always mean a reduced closed subscheme.

The main result of this paper is the following.

Theorem 1.2. Let $\left\{P_{i}\right\}_{1 \leq i \leq r}$ and $\left\{Q_{j}\right\}_{1 \leq j \leq r}$ be two collections of Brauer-Severi surfaces (resp. conics) over $S$. Consider the following conditions.

(i) $\left\langle\left\{P_{i}\right\}\right\rangle=\left\langle\left\{Q_{j}\right\}\right\rangle$ in $\operatorname{Br}(S)$.

(ii) $\left[\prod P_{i}\right]=\left[\prod Q_{j}\right]$ in $M(S)$.

(iii) $\prod P_{i}$ and $\prod Q_{j}$ are birational.

Then $(i) \Rightarrow($ ii $)$. If $S$ is reduced, then $(i) \Rightarrow($ iii $)$. If $S$ is a separated regular scheme, then $(i) \Longleftrightarrow($ iii). If $S$ is a separated regular scheme with characteristic zero generic points, then (i), (ii) and (iii) are equivalent.

This result has been inspired by 2, where relations between products of conics in the Grothendieck ring were studied for the first time. The above theorem was proved in 2 for conics in the case when $S=\operatorname{Spec}(k)$, where $k$ is a number field or function field of an algebraic surface over $\mathbb{C}$.

The proof presented here is by induction on $r$. Working over a general noetherian base scheme $S$ instead of a field enables us to run the induction more smoothly.

Received by the editors December 29, 2006, and, in revised form, June 23, 2007, and November 30, 2007.

2000 Mathematics Subject Classification. Primary 14E05, 14M99; Secondary 14J25.

Key words and phrases. Brauer-Severi surfaces, Grothendieck ring, birational maps. 
Recall the following conjecture of Amitsur.

Conjecture 1.3 ([1]). Let $k$ be a field and let $P$ and $Q$ be $n$-dimensional BrauerSeveri varieties over $k$. Then $P$ is birational to $Q \Longleftrightarrow P$ and $Q$ generates the same subgroup in $\mathrm{Br}(k)$.

This conjecture is still unknown in general; however, the following special cases are known.

(1) $P$ is split by a cyclic extension (which is always true if $k$ is a local or global field) (see [1]).

(2) $\operatorname{index}(P)<\operatorname{dim}(P)+1$ (see [4]).

(3) $P=-Q$ in $B r(k)$ (this proves the conjecture for Brauer-Severi surfaces) (see 44).

(4) $P=2 Q$ in $\operatorname{Br}(k)$ (see [5]).

Remark 1.4. In addition to Brauer-Severi surfaces and conics, the proof of Theorem 1.2 presented here also works for Brauer-Severi varieties of prime index if one assumes Amitsur's conjecture for this case and an analogue of Theorem 3.3 .

\section{Preliminaries on the Grothendieck Ring}

2.1. Grothendieck ring. Let $S$ be any scheme. Let $M(S)$ denote the free abelian group generated on isomorphism classes of reduced finite type $S$-schemes modulo the relations

$$
[X]=[U]+[Z]
$$

where $X$ is a reduced $S$-scheme and $U \subset X$ is an open subset with complement $Z$ (with reduced scheme structure). For any $S$-scheme $X$, we will use the notation $[X]_{S}$ or just $[X]$ to denote the class of $X^{\text {red }}$ in $M(S)$. For $S$-schemes $X, Y$ define

$$
[X]_{S} \cdot[Y]_{S}=\left[\left(X \times_{S} Y\right)\right]_{S} .
$$

This makes $M(S)$ into a commutative and associative ring with $[S]$ being the identity in this ring. $M(S)$ is called the Grothendieck ring of finite type $S$-schemes. Notice that $M(S)$ depends only on the reduced structure of $S$.

2.2. $f^{*}$ and $f_{*}$. Given any morphism $f: T \rightarrow S$, there is a functorial ring homomorphism $f^{*}: M(S) \rightarrow M(T)$ induced by base extension $X \rightarrow X \times_{S} T$. Moreover, if $f$ is itself of finite type, one also has a morphism of $M(S)$-modules $f_{*}: M(T) \rightarrow M(S)$ induced by considering any $T$-scheme as an $S$-scheme via $f$.

Suppose we have a filtered inverse system of schemes $\left\{S_{i}\right\}_{i \in I}$ such that the

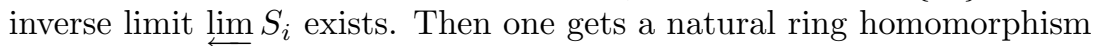

$$
\underset{\lim }{\longrightarrow} M\left(S_{i}\right) \rightarrow M\left(\lim _{\longleftarrow} S_{i}\right)
$$

The following special case is of special interest.

Proposition 2.3. Let $S$ be an integral scheme. Let $\{U\}_{U \subset S}$ be the (filtered) inverse system of nonempty open sets of $S$. Let $K$ be the function field of $S$. Then the natural ring homomorphism $\lim _{U \subset S} M(U) \rightarrow M(\operatorname{Spec}(K))$ is an isomorphism.

Proof. Any finite type $K$-scheme $X_{K}$ is the generic fibre of some finite type $U$ scheme $X_{U}$ for some nonempty open set $U$ of $S$. This shows that the above map is surjective. After shrinking $U$ if necessary, any closed subscheme $Z_{K} \subset X_{K}$ can be realized as the generic fibre of a closed subscheme $Z_{U} \subset X_{U}$. This shows that the map is injective. 
Finally, we recall the following elementary proposition.

Proposition 2.4 (Well-known). Let $\mathcal{E}$ be a vector bundle on $S$ of rank $n+1$. Then $[\operatorname{Proj}(\mathcal{E})]=\left[\mathbb{P}_{S}^{n}\right]$ in $M(S)$.

Proof. Let $S=\bigcup_{i=1}^{m} U_{i}$ be an open cover such that $\mathcal{E}_{\mid U_{i}}$ is trivial for each $i$. We now proceed by induction on $m$. If $m=1, \mathcal{E}$ is a trivial vector bundle and the statement is obvious. For $m>1$, let $S^{\prime}=\bigcup_{i=1}^{m-1} U_{i}$. Then by induction $\left[\operatorname{Proj}\left(\mathcal{E}_{\mid S^{\prime}}\right)\right]=\left[\mathbb{P}_{S^{\prime}}^{n}\right]$. Since $\mathcal{E}$ is trivial on $U_{m}$ and $S^{\prime} \cap U_{m}$, we also have $\left[\operatorname{Proj}\left(\mathcal{E}_{\mid U_{m}}\right)\right]=\left[\mathbb{P}_{U_{m}}^{n}\right]$ and $\left[\operatorname{Proj}\left(\mathcal{E}_{\mid S^{\prime} \cap U_{m}}\right)\right]=\left[\mathbb{P}_{S^{\prime} \cap U_{m}}^{n}\right]$. The result now follows from the following equalities in $M(S)$ :

$$
\begin{aligned}
{[\operatorname{Proj}(\mathcal{E})] } & =\left[\operatorname{Proj}\left(\mathcal{E}_{\mid S^{\prime}}\right)\right]+\left[\operatorname{Proj}\left(\mathcal{E}_{\mid U_{m}}\right)\right]-\left[\operatorname{Proj}\left(\mathcal{E}_{\mid S^{\prime} \cap U_{m}}\right)\right] \\
{\left[\mathbb{P}_{S}^{n}\right] } & =\left[\mathbb{P}_{S^{\prime}}^{n}\right]+\left[\mathbb{P}_{U_{m}}^{n}\right]-\left[\mathbb{P}_{S^{\prime} \cap U_{m}}^{n}\right] .
\end{aligned}
$$

\section{The Cremona map}

3.1. The Cremona map. Let $K$ be a field. Let us recall the following well-known birational map from $\mathbb{P}_{K}^{2}$ to itself:

$$
\phi: \mathbb{P}_{K}^{2} \rightarrow \mathbb{P}_{K}^{2} \quad[X, Y, Z] \rightarrow[Y Z, X Z, X Y] .
$$

$\phi$ can be defined everywhere on $\mathbb{P}_{K}^{2}$ outside the reduced closed subscheme

$$
B=\{[1,0,0],[0,1,0],[0,0,1]\} .
$$

Let $X \stackrel{p}{\rightarrow} \mathbb{P}_{K}^{2}$ be the blowup of $\mathbb{P}_{K}^{2}$ with center $B$. Then $\phi$ defines a morphism $X \stackrel{q}{\rightarrow} \mathbb{P}_{K}^{2}$ such that the following diagram commutes.

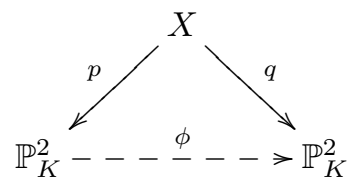

One can check that $q: X \rightarrow \mathbb{P}_{K}^{2}$ is itself is the blowup of $\mathbb{P}_{K}^{2}$ again with center $B$.

The following result was essentially proved in [4].

Theorem 3.2. Let $P$ and $Q$ be Brauer-Severi surfaces over a field $K$. Assume that $P=2 Q$ in $B r(K)$. Then there exists a birational map $\phi: P \rightarrow Q$ which after going to $\bar{K}$ (the separable closure of $K$ ) is isomorphic to the Cremona map.

Theorem 3.3. Let $K$ be any field and let $P$ and $Q$ be Brauer-Severi surfaces which generate the same subgroup in $\operatorname{Br}(K)$. Then $[P]=[Q]$ in $M(K)$.

Proof. Let $\phi: P \rightarrow Q$ be a map as guaranteed by Theorem 3.2 Let $B$ (resp. $B^{\prime}$ ) be the base locus of the map $\phi$ (resp. $\phi^{-1}$ ). Without loss of generality we may assume that $P$ defines a nontrivial class in $\operatorname{Br}(K)$ and thus has no $K$-point. Then $B$ (resp. $B^{\prime}$ ) is a closed $L$-point (resp. $L^{\prime}$-point) of $P$ (resp. $Q$ ) for some degree 3 separable field extension $L / K$ (resp. $L^{\prime} / K$ ). We claim that $L / K$ and $L^{\prime} / K$ are isomorphic field extensions. Let $X$ be the blowup of $P$ at $B$. Then we have the following Hironaka hut.

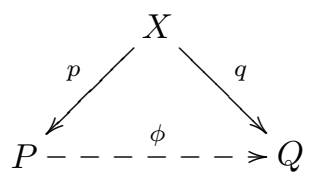


Here $q$ is the blowup of $Q$ at $B^{\prime}$ (see Section 3.1). To show that $L / K$ and $L^{\prime} / K$ are isomorphic it is enough to show that $B^{\prime} \times_{K} \operatorname{Spec}(L)$ has an $L$-point. But after base extending to $L, P_{L}=P \times_{K} L \cong \mathbb{P}_{L}^{2}$ and $p$ is the blowup of three $L$-points of $P_{L}$, say $x, y, z$. Let $L_{x y}$ be the unique line in $P_{L}$ joining $x$ and $y$ and similarly for $L_{y z}, L_{x z}$. Then the birational transform of $L_{x y} \cup L_{y z} \cup L_{x z}$ is the exceptional locus of $q$. Thus the image, $B^{\prime} \times{ }_{K} \operatorname{Spec}(L)$, of this exceptional locus is the disjoint union of 3 points. This proves the claim.

Thus as $K$-varieties, the exceptional locus of $p$ (resp. $q$ ) is isomorphic to $\mathbb{P}_{L}^{1}$. Thus $[P]=[X]-\left[\mathbb{P}_{L}^{1}\right]+[\operatorname{Spec}(L)]=[Q]$.

\section{Proof of the MAIN THEOREM}

For any morphism $f: T \rightarrow S$ and any $S$-scheme $X$, let $X_{T}=X \times_{S} T$.

Lemma 4.1. Let $X$ be any finite type $S$-scheme. Let $P$ be a Brauer-Severi scheme over $S$ of relative dimension n. Assume that the class of $P$ in $B r(S)$ lies in the kernel of $\operatorname{Br}(S) \rightarrow \operatorname{Br}(X)$. Then

$$
[X \times P]_{S}=\left[\mathbb{P}_{X}^{n}\right]_{S} .
$$

Proof. It is enough to prove $[X \times P]_{X}=\left[\mathbb{P}_{X}^{n}\right]_{X}$ since the required equality can then by obtained by using the natural map $M(X) \rightarrow M(S)$. But by assumption, the class represented by $X \times P$ in $B r(X)$ is zero. Hence there exists a vector bundle $\mathcal{E}$ on $X$ such that $X \times P$ is isomorphic to $\operatorname{Proj}(\mathcal{E})$ as $X$-schemes. The result now follows from Proposition 2.4

Proof of Theorem 1.2. In order to avoid unnecessary repetition, we will only prove the theorem for Brauer-Severi surfaces. We proceed by induction on $r$.

$(i) \Rightarrow(i i)$ :

Step 1. One can quickly reduce to proving the statement in the case when $S$ is integral. Let $S=S_{1} \cup S_{2}$ be the decomposition of $S$ into two closed subschemes. Then for any $S$-scheme $X$ of finite type, we have

$$
[X]_{S}=\left[X_{S_{1}}\right]_{S}+\left[X_{S_{2}}\right]_{S}-\left[X_{S_{12}}\right]_{S} \text { where } S_{12}=S_{1} \cap S_{2} .
$$

Hence, by noetherian induction and the above formula, it is enough to prove the theorem in the case when $S$ is irreducible. Moreover, the natural ring homomorphism $M(S) \rightarrow M\left(S^{r e d}\right)$ is an isomorphism. Thus, we may assume $S$ is integral.

Step $2(r=1)$. Let $P / S$ and $Q / S$ be two Brauer-Severi surfaces. Let $K$ be the function field of $S$. Then by Theorem 3.3 .

$$
\left[P_{K}\right]=\left[Q_{K}\right] \quad \text { in } M(K),
$$

By Proposition 2.3, there exists a nonempty open set $U$ of $S$ such that

$$
\left[P_{U}\right]=\left[Q_{U}\right] \quad \text { in } M(U) .
$$

Let $Z$ be the complement of $U$. By noetherian induction $\left[P_{Z}\right]=\left[Q_{Z}\right]$. Since $[P]=\left[P_{U}\right]+\left[P_{Z}\right]$ and similarly for $[Q]$, we get that $[P]_{S}=[Q]_{S}$.

Step 3. Suppose the dimension of $\left\langle\left\{P_{i}\right\}\right\rangle$ (and hence also of $\left\langle\left\{Q_{j}\right\}\right\rangle$ ) as an $\mathbb{F}_{3}$ vector space is strictly less than $r$. Then without loss of generality we may assume that 
the class of $P_{r}$ is contained in the subgroup generated by $\left\{P_{i}\right\}_{1 \leq i \leq r-1}$. Then by Lemma 4.1

and similarly for the $Q_{i}$ 's. By induction,

$$
\left[\prod_{i} P_{i}\right]=\left[\prod_{i \leq r-1} P_{i} \times \mathbb{P}_{S}^{2}\right]
$$

$$
\left[\prod_{1 \leq i \leq r-1} P_{i}\right]=\left[\prod_{1 \leq j \leq r-1} Q_{j}\right]
$$

which implies

$$
\left[\prod_{1 \leq i \leq r} P_{i}\right]=\left[\prod_{1 \leq i \leq r-1} P_{i} \times \mathbb{P}_{S}^{2}\right]=\left[\prod_{1 \leq j \leq r-1} Q_{j} \times \mathbb{P}_{S}^{2}\right]=\left[\prod_{1 \leq j \leq r} Q_{j}\right]
$$

Hence, it is enough to prove the theorem under the extra assumption that the dimension of $\left\langle\left\{P_{i}\right\}_{1 \leq i \leq r}\right\rangle$ as an $\mathbb{F}_{3}$ vector space is $r$.

Step 4. Since the class of $Q_{1}$ is in the subgroup generated by $P_{i}$ 's in $\operatorname{Br}(S)$, we have the following equation in $\operatorname{Br}(S)$ :

$$
Q_{1}=\sum a_{i} P_{i}, \quad a_{i} \in \mathbb{F}_{3}
$$

By Step 3, at least one of the $a_{i}$ 's is nonzero. Without loss of generality we may assume $a_{1} \neq 0$. Thus, $Q_{1}=a_{1} P_{1}+\sum_{i \geq 2} a_{i} P_{i}$ in $\operatorname{Br}(S)$. We first claim that

$$
\left[Q_{1} \times \prod_{2 \leq i \leq r} P_{i}\right]_{S}=\left[P_{1} \times \prod_{2 \leq i \leq r} P_{i}\right]_{S} \text {. }
$$

Put $Y=\prod_{2 \leq i<r} P_{i}$. Now $Q_{1} \times Y$ and $P_{1} \times Y$ generate the same subgroup in $\operatorname{Br}(Y)$. Hence, by Step 2, $\left[Q_{1} \times Y\right]_{Y}=\left[P_{1} \times Y\right]_{Y}$. The claim now follows by using the push-forward map $M(Y) \rightarrow M(S)$. Now to prove the theorem it is enough to show

$$
\left[Q_{1} \times \prod_{2 \leq i \leq r} P_{i}\right]_{S}=\left[Q_{1} \times \prod_{2 \leq i \leq r} Q_{i}\right]_{S} .
$$

But the subgroup generated by $\left\{P_{i}\right\}_{2 \leq i \leq r}$ in $\operatorname{Br}\left(Q_{1}\right)$ is the same as the subgroup generated by $\left\{Q_{i}\right\}_{2 \leq i \leq r}$. Hence by induction on $r$ we have

$$
\left[Q_{1} \times \prod_{2 \leq i \leq r} P_{i}\right]_{Q_{1}}=\left[Q_{1} \times \prod_{2 \leq i \leq r} Q_{i}\right]_{Q_{1}} .
$$

Again, the claim follows by using the push-forward map $M\left(Q_{1}\right) \rightarrow M(S)$.

$(i) \Rightarrow(i i i)$ ( $S$ is reduced). As in the proof of $(i) \Rightarrow(i i)$, we proceed by induction on $r$. One first reduces the proof to the case when $S$ is integral. Then by noetherian induction and the known result for the case when $S$ is the spectrum of a field we prove the case $r=1$. After a possible re-indexing, one then proves $P_{1} \times \prod_{2 \leq i \leq r} P_{i}$ is birational to $Q_{1} \times \prod_{2 \leq i \leq r} Q_{i}$ by first comparing $P_{1} \times \prod_{2 \leq i \leq r} P_{i}$ and $Q_{1} \times \prod_{2 \leq i \leq r} P_{i}$ and then comparing $Q_{1} \times \prod_{2 \leq i \leq r} P_{i}$ and $Q_{1} \times \prod_{2 \leq i \leq r} Q_{i}$. Since the argument is very similar to the one above, we leave the details to the reader. 
$($ iii $) \Rightarrow(i)(S$ is a separated regular scheme). Without loss of generality we may assume $S$ is connected. Let $K$ be the function field of $S$. Since $S$ is regular, $\operatorname{Br}(S) \rightarrow \operatorname{Br}(K)$ is injective. Thus, in order to prove $(i i i) \Rightarrow(i)$ and $(i i) \Rightarrow(i)$, we may replace $S$ by $\operatorname{Spec}(K)$. The kernel of $B r(K) \rightarrow B r\left(\prod P_{i}\right)$ is equal to $\left\langle\left\{P_{i}\right\}\right\rangle$. Moreover, this kernel depends only on the stable birational class of $\prod P_{i}$. This proves that $(i i i) \Rightarrow(i)$.

If $K$ is of characteristic zero, then $(i i) \Rightarrow(i)$ follows from the fact that any two smooth projective varieties having the same image in $M(K)$ are stably birational (see [3]).

\section{ACKNOWLEDGEMENTS}

I thank my advisor, János Kollár, for his encouragement and useful discussions. I also thank Chenyang $\mathrm{Xu}$ for useful comments and discussions. I thank Daniel Krashen for pointing out an error in the previous version.

\section{REFERENCES}

1. Amitsur, S. A., Generic splitting fields of central simple algebras, Ann. of Math (2) 62 (1955), 8-43. MR0070624 (17:9d)

2. Kollár, J., Conics in the Grothendieck ring, Adv. Math. 198 (2005), no. 1, 27-35. MR.2183248 (2006k:14064)

3. Larsen, M., Lunts, V.A., Motivic measures and stable birational geometry. Mosc. Math. J. 3 (2003), no. 1, 85-95. MR.1996804(2005a:14026)

4. Roquette, P., On the Galois cohomology of the projective linear group and its applications to the construction of generic splitting fields of algebras, Math. Ann. 150 (1963), 411-439. MR0154888 (27:4832)

5. Tregub, S. L., Birational equivalence of Brauer-Severi manifolds, Uspekhi Mat. Nauk 46 (1991), 217-218; English translation in Russian Math. Surveys 46 (1991), 229. MR.1164209(93d:14035)

Department of Mathematics, Princeton University, Princeton, New Jersey 08544

E-mail address: amit@math.princeton.edu

Current address: School of Mathematics, Tata Institute of Fundamental Research, Homi

Bhabha Road, Colaba, Mumbai 400005, India

E-mail address: amit@math.tifr.res.in 